\title{
The Implications of Several Forms of Programmed Necrosis for Cancer Therapy \\ Byeong Mo Kim*
}

Severance Integrative Research Institute for Cerebral and Cardiovascular Diseases (SIRIC), Yonsei University College of Medicine, 50 Yonsei-ro, Seodaemun-gu, Seoul 120-752, Republic of Korea

\begin{abstract}
The dogma that apoptosis and autophagy are the sole forms of Programmed Cell Death (PCD) is no longer accepted, due to the recent discovery of programmed necrosis, a non-apoptotic, non-autophagic form of PCD. Necroptosis, parthanatos, pyroptosis, and ferroptosis are all forms of programmed necrosis, as these types of cell death dismantle the cell in an ordered fashion that is distinctly different from apoptosis or autophagy. Several key cellular mediators and events in these types of PCD have been discovered. Here, we discuss the basic characteristics, molecular pathways, and possible implications of these lesser-known types of PCD in cancer treatment modalities such as chemotherapy and radiotherapy. Because resistance to apoptosis is often responsible for cancer treatment failures, novel therapeutic strategies that can activate alternative cell death programs have great appeal. Understanding the underlying mechanisms of these types of PCD may facilitate the development of diverse therapeutic strategies, particularly against apoptosis-resistant cancers.
\end{abstract}

Keywords: Non-apoptotic or non-autophagic programmed cell death; Programmed necrosis; Necroptosis; Parthanatos; Pyroptosis; Ferroptosis; Cancer treatment

\section{Introduction}

Cell death occurs through programmed or non-programmed processes. Programmed cell death (PCD), typically referring to apoptosis and autophagy, is mediated by organized intracellular programs, and usually occurs in biological developmental processes or is involved in tumor progression and response to cancer therapeutics. Non-programmed cell death (non-PCD), typically referring to necrosis, is a non-physiological and unorganized process, and usually occurs following acute injury and infection. Recent research has defined a subset of non-apoptotic/non-autophagic cell death modes that are controlled by specific signaling pathways that are morphologically distinct from apoptosis or autophagy, involving membrane rupture and the release of cytoplasmic contents. The view that apoptosis and autophagy are the sole means by which the cell can undergo PCD should be changed in light of the discoveries of additional modes of PCD, namely, programmed necrosis. Also termed regulated necrosis, programmed necrosis distinguishes the processes carried out by a specific program of genetically encoded cellular apparatuses from classical necrosis, which is carried out in an accidental and passive way. With the discovery of key mediators of necrotic death, such as receptorinteracting serine/threonine protein (RIP) kinases and poly (ADPribose) polymerases (PARPs), the concept of programmed necrosis is gaining ground.

Although the precise mechanisms responsible for programmed necrosis remain largely elusive, studies within the past 7 to 8 years have substantially increased our understanding of the process. Recent in vivo studies suggest that programmed necrosis is important for both physiological and pathological processes [1]. Programmed necrosis has unique features. Several modes of programmed necrosis have been identified, and their key regulators and events, such as RIP kinases, necrosomes, cyclophilin $\mathrm{D}$, inhibition of caspases, mitochondrial fission, Bmf, PARP, Bax, apoptosis-inducing factor (AIF), Bcl-2 nineteen-kilodalton interacting protein 3 (BNIP3), Nix, NADPH oxidases, cathepsins, and calpains, have been identified as potential signaling components of the necrotic program. Necroptosis is at present the major and most well understood form of regulated necrosis. The necrosome complex, composed of RIP1/3 and mixed lineage kinase domain-like protein (MLKL) and regulated by RIP1 and RIP3 kinases, is the primary cellular complex that regulates necroptosis, a major form of programmed necrosis. The necrosome mediates upstream cell death receptors and downstream executing molecules and events, such as reactive oxygen species (ROS) bursts, plasma membrane permeabilization, and cytosolic ATP reduction [2]. The cellular pathways involving PARP activation and AIF translocation from the mitochondria to the nucleus contribute to parthanatos, a second major programmed necrosis pathway. In addition to necroptosis and parthanatos, other types of programmed necrosis, such as pyroptosis, ferroptosis, oxytosis, pyronecrosis, ETosis, and NETosis have also been identified. Table 1 outlines various modes of programmed necrosis and their key events and regulatory machinery in comparison with other cell death modes.

\section{Literature Review}

In this review, we discuss each type of programmed necrosis and their therapeutic implications for cancer treatment purposes. Due to their relatively profound characterization and findings, we primarily focus on necroptosis and parthanatos.

\section{Necroptosis}

The most well-studied form of programmed necrosis is necroptosis which is typically activated in response to death receptor activation.

*Corresponding author: Dr. Byeong Mo Kim, Severance Integrative Research Institute for Cerebral and Cardiovascular Diseases (SIRIC), Yonsei University College of Medicine, 50 Yonsei-ro, Seodaemun-gu, Seoul 120-752, Republic of Korea, Tel: 82 2-2228-0789; E-mail: bkim2@yuhs.ac

Received August 01, 2017; Accepted September 25, 2017; Published September 27, 2017

Citation: Kim BM (2017) The Implications of Several Forms of Programmed Necrosis for Cancer Therapy. J Cancer Sci Ther 9: 630-635. doi:10.4172/1948 5956. 1000484

Copyright: (c) $2017 \mathrm{Kim}$ BM. This is an open-access article distributed under the terms of the Creative Commons Attribution License, which permits unrestricted use, distribution, and reproduction in any medium, provided the original author and source are credited. 


\begin{tabular}{|c|c|c|c|c|c|c|c|}
\hline \multirow[t]{3}{*}{ Variables } & \multicolumn{6}{|c|}{ Programmed } & \multirow{3}{*}{$\begin{array}{c}\begin{array}{c}\text { Non- } \\
\text { programmed }\end{array} \\
\text { Necrosis }\end{array}$} \\
\hline & \multirow[t]{2}{*}{ Apoptosis } & \multirow[t]{2}{*}{ Autophagy } & \multicolumn{4}{|c|}{ Programmed necrosis } & \\
\hline & & & Necroptosis & Parthanatos & Pyroptosis & Ferroptosis & \\
\hline \multirow[t]{3}{*}{ Trigger } & Death receptor & Starvation & \multirow{3}{*}{$\begin{array}{c}\text { Death receptor } \\
\text { activation and inhibition } \\
\text { of caspase-8 }\end{array}$} & Genotoxic stress & \multirow[t]{3}{*}{ Inflammasomes } & $\begin{array}{l}\text { Experimental } \\
\text { compounds }\end{array}$ & Infection \\
\hline & \multirow[t]{2}{*}{ Intrinsic } & \multirow[t]{2}{*}{ Rapamycin } & & \multirow[t]{2}{*}{ Excitotoxicity } & & \multirow[t]{2}{*}{ Clinical drugs } & Toxins \\
\hline & & & & & & & Trauma \\
\hline $\begin{array}{l}\text { Inflammatory } \\
\text { response }\end{array}$ & No & Yes & Yes & Yes & Yes & Yes & Yes \\
\hline \multirow[t]{4}{*}{ Aspects } & $\begin{array}{c}\text { Chromatin } \\
\text { condensation }\end{array}$ & Membrane blebbing & Mitochondrial swelling & $\begin{array}{c}\text { Chromatin } \\
\text { condensation }\end{array}$ & Cell swelling & $\begin{array}{l}\text { Increased } \\
\text { Membrane } \\
\text { density }\end{array}$ & $\begin{array}{l}\text { Mitochondrial } \\
\text { swelling }\end{array}$ \\
\hline & Nuclear fragmentation & Autophagic vacuoles & Cell swelling & \multirow{3}{*}{$\begin{array}{c}\text { Nuclear } \\
\text { fragmentation }\end{array}$} & Membrane rupture & \multirow{3}{*}{$\begin{array}{c}\text { Small } \\
\text { mitochondria }\end{array}$} & Cell swelling \\
\hline & Apoptotic body & \multirow{2}{*}{$\begin{array}{l}\text { Increased lyssomal } \\
\text { activity }\end{array}$} & \multirow[t]{2}{*}{ Membrane rupture } & & \multirow{2}{*}{$\begin{array}{c}\text { Maintained } \\
\text { Mitochondrial integrity }\end{array}$} & & \multirow{2}{*}{$\begin{array}{l}\text { Membrane } \\
\text { rupture }\end{array}$} \\
\hline & Membrane blebbing & & & & & & \\
\hline $\begin{array}{l}\text { Key } \\
\text { regulators }\end{array}$ & $\begin{array}{l}\text { Cytochrome c } \\
\text { Caspases }\end{array}$ & $\begin{array}{l}\text { Atg proteins } \\
\text { Beclin-1 }\end{array}$ & $\begin{array}{l}\text { RIP } 1 / 3 \\
\text { MLKL }\end{array}$ & $\begin{array}{l}\text { PARPs } \\
\text { AlF }\end{array}$ & $\begin{array}{c}\text { Caspase-1/4/5/11 } \\
\text { Gasdermin D }\end{array}$ & GPX4 & - \\
\hline
\end{tabular}

Table 1: Overview and comparison of various cell death pathways.

The term necroptosis arose from its unique characteristics combining apoptotic and necrotic features. Like apoptosis, necroptosis is carried out by a series of organized cellular components. As in necrosis, the ultimate stage of a necroptotic process is the swelling and rupture of the cell membrane, releasing damage-associated molecular patterns (DAMPs). Recently, a number of studies have provided new insights into the molecular regulation of necroptosis.

Although ligands such as Fas ligand (FasL) and tumor necrosis factor alpha (TNFa) stimulate apoptosis, they also kill cells in a nonapoptotic manner with a necrotic morphology in other situations. This death receptor-specific necrotic death was designated necroptosis [3]. Necroptosis mainly occurs downstream of death receptors and is activated by the ligation of death ligands such as TNFa, TNF-related apoptosis-inducing ligand (TRAIL), and FasL, to their cognate receptors, including the TNF receptor superfamily members TNF receptor (TNFR), TRAIL receptor (TRAILR), TNF-related weak inducer of apoptosis (TWEAK), lymphotoxic beta (LT $\beta$ ) receptor, and Fas. Ligand-receptor binding results in the assembly of a supramolecular complex composed of caspase-8, the adaptor protein Fas-associated death domain (FADD), and RIP1/3. In this type of cell death, RIP1 plays a role in the branch point between survival and death [4]. When RIP1 is ubiquitinated, it induces survival signals. In contrast, when RIP1 is deubiquitinated, it induces apoptotic or necrotic signals depending on the action of caspase- 8 . Under conditions in which caspase- 8 function is favored, active caspase- 8 cleaves RIP $1 / 3$ and inactivates them, triggering apoptosis. However, when caspases are inactivated, the proteolytic cleavage of RIP1 and RIP3 is inhibited, thereby leading to RIP1/3 activation and resultant necroptosis. Induction of necroptosis can also occur independently of death receptor pathways through the engagement of pattern recognition receptors, such as interferon receptors and Toll-like receptors (TLR3 and TLR4), and viral expression of RIP homotypic interaction motif (RHIM)-containing proteins. In addition, genotoxic or oxidative stress induced by anticancer drugs, ionizing radiation, photodynamic therapy, ischemia-reperfusion injury, or calcium overload have been described as initiators of necroptosis. A variety of stimuli have been implicated as initiators of necroptosis, and the list of necroptotic triggers is constantly growing.

Necroptosis is initiated by the necroptosome, a protein complex that contains RIP1 and RIP3. RIP1 kinase activity is required for death receptor- and caspase inhibitor-mediated necroptosis in both murine and human cells. RIP3 interacts with RIP1 under necrotic cell death conditions and is an essential downstream partner for RIP1 in necroptosis. Although some necroptosis inducers may bypass RIP1 to directly activate RIP3 [5], considering that necrostatin 1 inhibits both RIP1 and necroptosis, RIP1 may be essential for the cellular necroptotic program. Necroptosis requires the inactivation of caspase-8 [6], which leads to the activation of RIPK1 and RIPK3. Necroptosis is also linked to rapid mitochondrial dysfunction and excessive ROS production. Although the mitochondrial permeability transition (MPT) pore is thought to contribute to both apoptotic and necrotic cell death, the MPT pore may be more pro-necrotic than pro-apoptotic. It is increasingly suggested that while mitochondrial outer membrane permeabilization (MOMP) is preferentially involved in apoptosis, the mitochondrial permeability transition (MPT) is involved in necroptosis. MPT pore opening and loss of mitochondrial membrane potential (MMP) trigger ROS release, which can then contribute to necroptosis. However, under certain situations, ROS and c-Jun N-terminal kinase (JNK) may also be involved upstream of mitochondria, triggering MMP loss and subsequent necroptosis.

Historically, the most well-studied mode of necroptotic cell death is TNFa-mediated necroptosis. In necroptotic signaling, TNFa binding to TNFR1 induces the formation of a membrane-bound pro-survival complex, which includes TNFR-associated death domain (TRADD), RIP1, and several ubiquitin E3 ligases, including cellular inhibitor of apoptosis proteins (cIAPs). In this complex, RIP1 is polyubiquitinated by cIAP1 and 2. Lysine 63 ubiquitination of RIP1 contributes positively to TNFa-induced survival signaling by facilitating the recruitment of kinases that activate the nuclear factor kappa B (NF- $\kappa \mathrm{B})$ and mitogenactivated protein kinase (MAPK) signaling pathways [4]. Ubiquitinated RIP1 also inhibits the formation and activation of RIP1-dependent apoptotic and necroptotic complexes, which is associated with cell survival by preventing RIP1 from interacting with the apoptotic or necrotic complexes. Cylindromatosis-mediated deubiquitination of RIP1 shifts cell fate from survival to death through the repression of NF- $\mathrm{kB}$ activation and through interactions with the proapoptotic machinery, such as caspase- 8 . Activated caspase- 8 cleaves and inactivates RIP1 and RIP3 to block necroptosis and induce apoptosis. However, under certain conditions, such as caspase inhibition, RIP1 and its sister kinase RIP3 are not cleaved, and form the necrosomal complex. Therefore, caspase-8-mediated degradation of RIP1 may represent one of the major molecular switches between apoptosis and necroptosis. Although the exact relationship between RIP1 and RIP3 
remains to be clarified, RIP1 and RIP3 phosphorylate and activate each other [7-9]. Both RIP1 and RIP3 contain kinase domains, but RIP3 lacks the death domain typically involved in the recruitment of proteins to death receptors. RIP1 interacts with and recruits RIP3 through a homotypic interaction motif (RHIM) at the C-terminal end of the intermediate domain contained within both proteins [10], resulting in their mutual phosphorylation.

Stable formation of the necrosome, the complex containing RIP1, RIP3, and associated proteins, depends on RIP1 kinase activity $[7,8]$. RIP1 kinase activity is also responsible for RIP3 phosphorylation. RIP1-induced RIP3 phosphorylation results in RIP3 oligomerization, which is necessary for its activation. MLKL is a well-identified substrate of RIP3. The interaction between RIP3 and MLKL is dependent upon the kinase activity of RIP3 and its phosphorylation at Ser227 [11]. RIP3 phosphorylates MLKL at both Thr357 and Ser358, and these phosphorylation events are required for MLKL action [11]. Phosphorylated MLKL trimerizes, translocates to the plasma membrane, and causes necrotic membrane permeabilization and disruption, which are crucial for necroptosis [12]. In the pro-necrotic necrosome complex, RIP3 interacts with several bioenergetic enzymes, including glycogen phosphorylase (PYGL), glutamate-ammonia ligase (GLUL), and glutamate dehydrogenase 1 (GLUD1), thereby enhancing metabolism accompanied by increased ROS production. The prevalence of the association of RIP3 with metabolic enzymes suggests that RIP3 may regulate energy production pathways associated with glycolysis and mitochondria. Upregulation of metabolic pathways may lead to the generation of ROS and impact mitochondrial function.

As in apoptosis, both mitochondria and lysosomes play pivotal roles in the propagation of the necroptotic death signal, and the main downstream target of the necroptosis pathway may be mitochondrial fragmentation. RIP1 or RIP3 localizes to the mitochondria in response to a necrotic stimulus $[13,14]$. Phosphoglycerate mutase family member 5 (PGAM5) and Drp1 proteins, as well as MLKL, may connect the necrosome to the mitochondria, thereby inducing mitochondrial fission, ROS generation, MMP loss, and resultant necrotic cell death in TNFa-treated cells. PGAM5 is recruited to the necrosome and activated, and activated PGAM5 recruits and activates Drp1 by dephosphorylating it. Additionally, given that the knockdown of Bmf, a BH3-only protein, blocks necroptosis induced by the combination of TNFa and caspase inhibition [15], Bmf is also one of the surprising candidates discovered as a mediator of TNFa-induced necroptosis. Although the exact molecular mechanisms by which Bmf promotes necroptosis versus apoptosis remain to be clarified, $\mathrm{Bmf}$ is a component of the core machinery of necroptosis. Necroptosis induced by TNFa occurs together with, and requires the production of, ROS. Mitochondrial and lysosomal ROS, as well as ROS generated by NADPH oxidases all contribute to necroptotic events. ROS and JNK activation are causatively related, and TNFa-induced necroptosis involves ROS generation and JNK activation. ROS- and JNK-dependent signaling events can result in the loss of MMP via the opening of the MPT pore, resulting in a loss of ATP production and mitochondrial rupture.

\section{Parthanatos}

Genotoxic stress-induced DNA damage causes necrotic cell death that is associated with an overstimulation of PARPs. Parthanatos, another type of programmed necrotic cell death, is characterized by, and dependent on, overactivation of the DNA damage-responsive nuclear PARP enzymes, and subsequent ATP depletion and translocation of AIF from the mitochondrial intermembrane space to the nucleus [1618]. Cyclophilin D, lysosomal proteases such as cathepsins, and non- lysosomal proteases such as calpains are also involved in parthanatos. This form of cell death has been implicated in a range of diseases, including Parkinson's disease, Alzheimer's disease, Huntington's disease, stroke, diabetes, and heart attacks. PARPs transfer the ADPribose moiety of $\mathrm{NAD}+$ to amino acids, thereby placing PAR polymers on various substrate proteins. PARP-mediated parthanatos is reportedly dependent on mitochondrial translocation of Bax [17]. Bax in turn induces parthanatos by releasing AIF from the mitochondria. Necrosis induced by hyperactivation of PARPs is simply due to metabolic catastrophe, in which the overactive PARPs use up cellular supplies of NAD+ and subsequent ATP. In addition to DNA damage-mediated necrotic death, PARPs also contribute to necrotic cell death initiated by TNF $\alpha$ and TRAIL. The parthanatic pathway not only interacts with the necrosome-associated pathway, but is also executed independently.

DNA damage-activated PARPs, particularly PARP1, drive necrotic cell death through multiple factors [19]. First, PARPs catalyze the polymerization of ADP-ribose units from donor NAD+ molecules on target proteins, resulting in NAD+ depletion [20]. In an effort to restore NAD+ levels, ATP is consumed, thereby depleting cellular ATP. Second, because NAD+ is an important cofactor for glycolysis, NAD+ depletion also inhibits glycolysis and prevents the formation of pyruvate and other downstream substrates of mitochondrial respiration pathways. Slowed glycolysis leads to a reduction in ATP production, as another ATP depletion mechanism. Third, the depletion of NAD+ promotes MPT pore opening-induced mitochondrial membrane depolarization, interfering with ATP production. Lastly, the PAR polymer produced by PARPs is itself cytotoxic by binding to AIF and inducing mitochondrial discharge of AIF [21]. Released AIF can cause DNA breaks, and AIF-mediated DNA degradation further activates PARPs, thereby initiating a vicious cycle. PARP-induced parthanatos is also dependent on the activation of $\mathrm{Ca} 2+$-activated calpains, which in turn impinge upon mitochondria [17]. Calpains favor the release of AIF from mitochondria, as well as the spillage of lysosomal cathepsins into the cytosol [22].

\section{Other Types of Programmed Necrosis}

Besides necroptosis and parthanatos, additional regulated forms of necrosis have been described, such as pyroptosis and feroptosis. Pyroptosis is a highly inflammatory form of programmed necrosis caused by various pathological stimuli, such as cancer, stroke, and heart attack, and is vital for controlling microbial infection. It is also triggered by cancer therapy. Inflammatory caspases, such as caspase- $1,-4,-5$, and -11 , are involved in this type of cell death. Pyroptosis is initiated by the formation and activation of multi-protein complex inflammasomes. Recently identified Gasdermin D, a component of inflammasomes and a substrate of inflammatory caspases, is also required for pyroptosis $[23,24]$.

Ferroptosis is another form of programmed necrotic cell death implicated in diverse degenerative diseases such as tissue ischemia/ reperfusion injury and Parkinson's disease. This form of cell death results from intracellular iron-dependent accumulation of lethal ROS and is characterized by lipid peroxidation. Ferroptotic cells display subtler morphological features, including smaller than normal mitochondria with condensed mitochondrial membrane density and outer mitochondrial membrane rupture. This form of cell death is caused by decreased activity of the lipid repair enzyme glutathione peroxidase 4 (GPX4), a key inhibitor of ferroptosis, and subsequent accumulation of lipid peroxides $[25,26]$. 


\section{The Implications of Programmed Necrosis for Cancer Therapy}

Given the rising significance of necrosis, especially programmed necrosis, in cancer, a better understanding of its implications in cancer development and maintenance is a prerequisite for the design of appropriate drugs. A growing body of evidence demonstrates that necroptosis is deregulated in cancer (Figure 1). For example, somatic mutations in RIP1, RIP3, and MLKL genes have been observed in human cancers. Single nucleotide polymorphisms in the RIP1 and RIP3 genes were detected in chronic myeloid leukemia and nonHodgkin's lymphoma patients, respectively [27,28]. Multiple cancers, including solid cancer cells, show suppressed RIP3 expression. RIP3 may be silenced by methylation in various cancer cell lines [29]. Necrotic regulators also seem to affect cancer prognosis. For example, low RIP3 levels are correlated with poor outcomes in colorectal and breast cancer patients [30-32]. RIP3 expression in the hematopoietic system may limit cancer invasion and metastasis. Likewise, reduced MLKL expression is significantly associated with decreased overall survival in gastric, ovarian, cervix, colon, and pancreatic cancers [33-37]. Thus, RIP3 and MLKL expression could potentially serve as prognostic biomarkers for these cancers. Overall, these studies suggest that programmed necrosis may play a tumor suppressive role in cancer. However, because the expression of necrotic players is elevated in some cancers, it is difficult to generalize the tumor suppressive activities of these regulators.

Therapeutic interventions that aim to induce apoptosis often face resistance arising from the activation of survival pathways. Thus, finding another cell death modality and more thoughtful combination therapies that simultaneously target alternative cell death and survival pathways is the main focus in cancer research. Triggering programmed necrosis could be useful when drugs fail to induce apoptosis. Targeting necroptosis and other types of programmed necrosis has been proposed as an emerging and attractive anti-tumor treatment strategy bypassing acquired apoptotic resistance and potentially switching on antitumor responses. In this section, we confine our description to necroptosisbased cancer therapy, due to its relatively well-documented findings.

Because a variety of cancer cell lines have necroptotic machinery and can undergo necroptosis, necroptotic therapy shows promise. Colorectal cancer cells and hematopoietic neoplasm cells seem

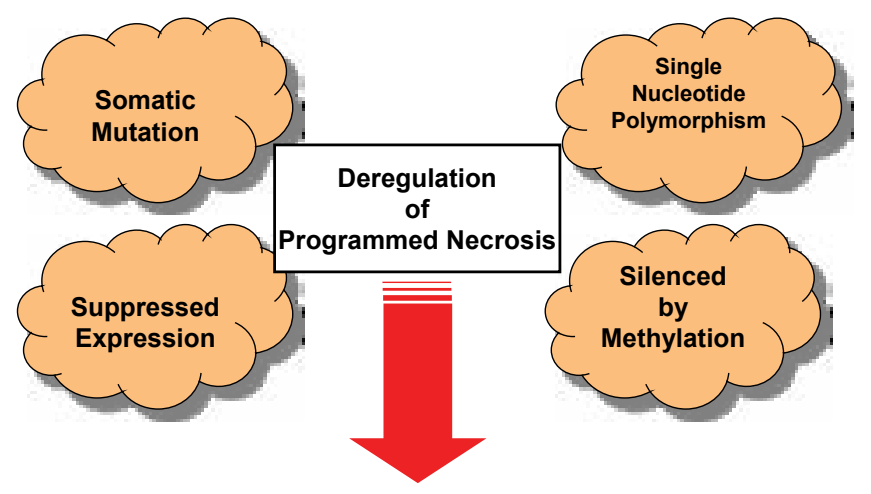

\section{Cancer Development}

Figure 1: Deregulation of components of the programmed necrotic pathway. particularly sensitive and responsive to necroptosis inducers [38]. However, other cancer cell lines, such as HeLa cervical cancer cells, HCT116 colorectal cancer cells, and OVCAR4 ovarian cancer cells, which lack effective necroptotic machinery, are unsuitable for necroptotic-based cancer therapy [38]. Furthermore, necroptosissensitive cells tend to only be responsive to a few specific necroptosis inducers rather than all necroptosis inducers. Necroptosis can be induced by cellular metabolic and genotoxic stress as well as ligandreceptor binding. A growing list of anticancer compounds with various mechanisms of action have been reported to initiate necroptosis in cancer cells. The strategies to induce necroptosis in cancer treatment are varied, and include classical necroptosis inducers, chemotherapeutic agents, ionizing radiation, and natural compounds (Figure 2).

Necroptosis is induced by chemotherapy, and is sometimes at least partially responsible for the toxicity of chemotherapeutic agents. RIP3 expression is required to sensitize a limited number of cancer cell lines to DNA-damaging agents in cancer chemotherapy. Necroptosis also plays an important role in radiation-induced cell death in endocrine cancers, including anaplastic thyroid cancers and adrenocortical cancers [39]. Treatment with necrostatin, a pharmacological inhibitor of RIP1, increases radioresistance in these cancer cell lines by enhancing DNA repair capacity. Moreover, necroptosis may be induced by natural compounds. Several reports suggest that shikonin, a major component of the Chinese herbal medicine zicao, kills cancer cells through the necroptosis pathway $[40,41]$.

Necroptosis triggers the anticancer immune response, which is another cancer-killing mechanism. Necrosis is believed to be more immunogenic than apoptosis. Combining caspase inhibitors with radiotherapy or chemotherapy reduces tumor growth due to the recruitment of immune cells. The ability of necroptosis to recruit and activate immune cells at tumor sites may increase the efficacy of the immune checkpoint blockade. Necroptotic cancer cells produce cytokines in a RIP1-dependent manner and release DAMPs that stimulate and attract dendritic cells to tumor sites. RIP3 induces the expression of cytokines that can activate natural killer $\mathrm{T}$ cells for

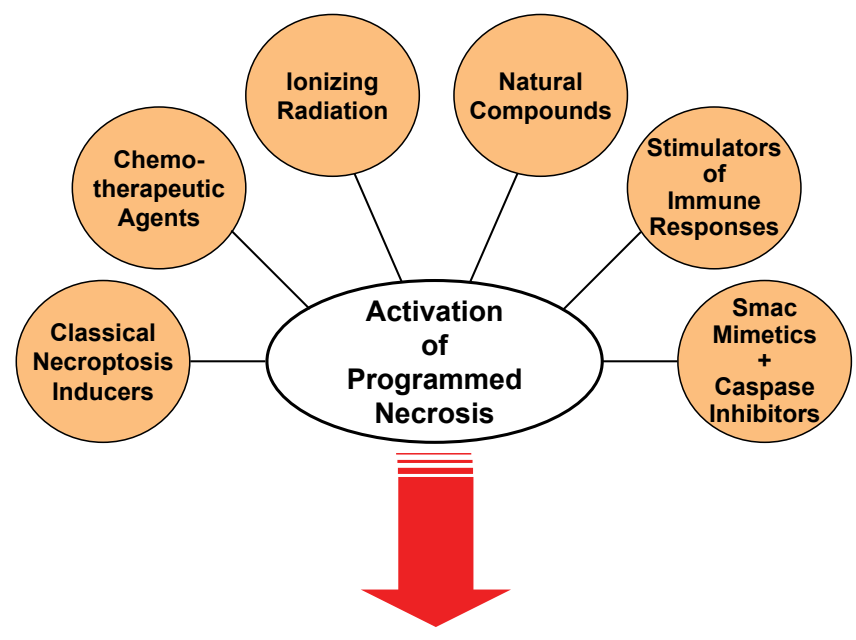

\section{Cancer Treatment}

Figure 2: Various anticancer strategies targeting molecules involved in the programmed necrotic pathway. 
anti-tumor activity. In contrast to RIP1 and RIP3, MLKL seems to be dispensable for cytokine synthesis $[42,43]$. Necroptosis may also be induced by autophagy. The BMI-1 inhibitor PTC-209 induces autophagy and upregulates RIP3, leading to necroptosis in ovarian cancer cells [44], indicating the existence of crosstalk between the autophagic and necroptotic machineries. Depletion of cIAP $1 / 2$ by second mitochondria-derived activator of caspase (Smac) mimetics deubiquitinates and unleashes RIP1 from its scaffolding pro-survival function and induces its recruitment to the ripoptosome, a complex that serves as a platform for regulating apoptosis or necroptosis. After that, RIP1 determines cell fate by regulating caspase-8-mediated apoptosis or promoting RIP3-MLKL-dependent necroptosis, which is dependent on the action of caspase- 8 . The specific induction of necroptosis requires the coordination of RIP kinase activation and caspase- 8 inhibition. Therefore, simultaneously targeting IAPs and caspase- 8 through genetic deletion or pharmacological inhibition using a combination of Smac mimetics and caspase inhibitors can specifically and unequivocally lead to necroptosis in cancer cells that have intact caspase- 8 function.

\section{Discussion and Perspectives}

Dysfunction and/or disruption of apoptotic machinery appear to be critical factors in intrinsic and acquired drug resistance in cancer cells. The failure of cancer cells to undergo apoptosis is a major problem in cancer treatment strategies. Therefore, novel therapeutic concepts to reactivate other cell death programs in human malignancies are needed. Programmed necrosis is a cell death modality that is controlled by defined signal transduction pathways. The deregulation of the programmed necrotic pathway observed in several cancers implicates it in cancer progression. Although little is known about the precise molecular targets in the programmed necrotic signaling pathway that are engaged by individual compounds, targeting programmed necrosis may be a plausible therapeutic intervention for activating another death pathway and/or boosting the immune system. Because programmed necrosis and apoptosis use distinct molecular pathways, triggering this regulated form of necrosis could be a particularly effective alternative method for eradicating apoptosis-resistant cancer cells. The requirement of caspase inhibition for effectively activating programmed necrosis represents a paradigm shift in cancer therapy, as most current therapeutic strategies aim to activate caspases. Because treatment resistance is among the most challenging currently unsolved problems in oncology, therapeutic induction of programmed necrosis may pave the way toward novel and more efficient treatment approaches.

\section{Conclusion}

It is still unknown whether programmed necrosis is a tolerable and feasible therapeutic target, or just a conceptual model. Although targeting programmed necrosis for cancer treatment presents several advantages over current strategies, a greater understanding of this type of therapeutic pathway is essential to assess the clinical achievability, as there is concern about this strategy due to the intrinsic or acquired defects of programmed necrotic machinery observed in many cancer cells. Two other concerns are whether programmed necrosis inducers selectively kill cancer cells without disturbing normal cells, and whether repetitive induction of programmed necrosis may lead to the development of chronic inflammatory diseases. A deeper understanding of the genetic and epigenetic context of programmed necrosis regulators will facilitate the development and administration of new mechanismbased therapeutics.

\section{Acknowledgement}

This work was supported by a grant from the Korea Healthcare Technol- ogy R and D Project, Ministry for Health and Welfare Affairs, Republic of Korea (HI08C2149) and by a faculty research grant from Yonsei University College of Medicine for 2016 (2016-32-0061).

\section{References}

1. Jouan-Lanhouet S, Riquet F, Duprez L, Vanden Berghe T, Takahashi N, et al (2014) Necroptosis, in vivo detection in experimental disease models. Sem Cel Dev Biol 35: 2-13.

2. Belizario J, Vieira-Cordeiro L, Enns S (2015) Necroptotic cell death signaling and execution pathway: Lessons from knockout mice. Media Inflamm 2015: 128076.

3. Degterev A, Huang Z, Boyce M, Li Y, Jagtap P, et al. (2005) Chemical inhibitor of nonapoptotic cell death with therapeutic potential for ischemic brain injury. Nat Chem Biol 1: 112-119.

4. Festjens N, Vanden Berghe T, Cornelis S, Vandenabeele P (2007) RIP1, a kinase on the crossroads of a cell's decision to live or die. Cell Death Differ 14: $400-410$.

5. Dillon CP, Weinlich R, Rodriguez DA, Cripps JG, Quarato G, et al. (2014) RIPK1 blocks early postnatal lethality mediated by caspase- 8 and RIPK3. Cell 157: 1189-1202.

6. Kroemer G, Galluzzi L, Vandenabeele P, Abrams J, Alnemri ES, et al. (2009) Classification of cell death: Recommendations of the nomenclature committee on cell death 2009. Cell Death Differ 16: 3-11.

7. He S, Wang L, Miao L, Wang T, Du F, et al. (2009) Receptor interacting protein kinase-3 determines cellular necrotic response to TNF-alpha. Cell 137: 11001111.

8. Cho YS, Challa S, Moquin D, Genga R, Ray TD, et al. (2009) Phosphorylationdriven assembly of the RIP1-RIP3 complex regulates programmed necrosis and virus-induced inflammation. Cell 137: 1112-1123.

9. Zhang DW, Shao J, Lin J, Zhang N, Lu BJ, et al. (2009) RIP3, an energy metabolism regulator that switches TNF-induced cell death from apoptosis to necrosis. Science 325: 332-336.

10. Sun X, Yin J, Starovasnik MA, Fairbrother WJ, Dixit VM (2002) Identification of a novel homotypic interaction motif required for the phosphorylation of receptorinteracting protein (RIP) by RIP3. J Biol Chem 277: 9505-9511.

11. Sun L, Wang H, Wang Z, He S, Chen S, et al. (2012) Mixed lineage kinase domain-like protein mediates necrosis signaling downstream of RIP3 kinase. Cell 148: 213-227.

12. Cai Z, Jitkaew S, Zhao J, Chiang HC, Choksi S, et al. (2014) Plasma membrane translocation of trimerized MLKL protein is required for TNFinduced necroptosis. Nat Cell Biol 16: 55-65

13. Kasof GM, Prosser JC, Liu D, Lorenzi MV, Gomes BC (2000) The RIP-like kinase, RIP3, induces apoptosis and NF-kappaB nuclear translocation and localizes to mitochondria. FEBS Lett 473: 285-291.

14. Temkin V, Huang Q, Liu H, Osada H, Pope RM (2006) Inhibition of ADP/ATP exchange in receptor-interacting protein-mediated necrosis. Mol Cell Biol 26 : 2215-2225.

15. Hitomi J, Christofferson DE, Ng A, Yao J, Degterev A, et al. (2008) Identification of a molecular signaling network that regulates a cellular necrotic cell death pathway. Cell 135: 1311-1323.

16. Yu SW, Wang H, Poitras MF, Coombs C, Bowers WJ, et al. (2002) Mediation of poly (ADP-ribose) polymerase-1-dependent cell death by apoptosis-inducing factor. Science 297: 259-263.

17. Moubarak RS, Yuste VJ, Artus C, Bouharrour A, Greer PA, et al. (2007) Sequential activation of poly (ADP-ribose) polymerase 1, calpains, and Bax is essential in apoptosis-inducing factor-mediated programmed necrosis. Mol Cell Biol 27: 4844-4862.

18. Artus C, Boujrad H, Bouharrour A, Brunelle MN, Hoos S, et al. (2010) AlF promotes chromatinolysis and caspase-independent programmed necrosis by interacting with histone H2AX. EMBO J 29: 1585-1599.

19. Morgan MJ, Liu ZG (2013) Programmed cell death with a necrotic-like phenotype. Biomole Concepts 4: 259-275.

20. van Wijk SJ, Hageman GJ (2005) Poly (ADP-ribose) polymerase-1 mediated caspase-independent cell death after ischemia/reperfusion. Free Radic Biol Med 39: 81-90. 
Citation: Kim BM (2017) The Implications of Several Forms of Programmed Necrosis for Cancer Therapy. J Cancer Sci Ther 9:630-635. doi:10.4172/19485956.1000484

21. Yu SW, Andrabi SA, Wang H, Kim NS, Poirier GG, et al. (2006) Apoptosisinducing factor mediates poly (ADP-ribose) (PAR) polymer-induced cell death. Proc Natl Acad Sci USA 103: 18314-18319.

22. Golstein P, Kroemer G (2007) Cell death by necrosis: Towards a molecular definition. Trends Biochem Sci 32: 37-43.

23. He WT, Wan HQ, Hu L, Chen P, Wang X, et al. (2015) Gasdermin D is an executor of pyroptosis and required for interleukin-1 beta secretion. Cell Res 25: $1285-1298$

24. Shi JJ, Gao WQ, Shao F (2017) Pyroptosis: Gasdermin-mediated programmed necrotic cell death. Trends Biochem Sci 42: 245-254.

25. Angeli JPF, Schneider M, Proneth B, Tyurina YY, Tyurin VA, et al. (2014) Inactivation of the ferroptosis regulator Gpx4 triggers acute renal failure in mice. Nat Cell Biol 16: 1180-U1120.

26. Yang WS, SriRamaratnam R, Welsch ME, Shimada K, Skouta R, et al. (2014) Regulation of Ferroptotic cancer cell death by GPX4. Cell 156: 317-331.

27. Cerhan JR, Ansell SM, Fredericksen ZS, Kay NE, Liebow M, et al. (2007) Genetic variation in 1253 immune and inflammation genes and risk of nonHodgkin lymphoma. Blood 110: 4455-4463.

28. Bruzzoni-Giovanelli H, Gonzalez JR, Sigaux F, Villoutreix BO, Cayuela JM, et al. (2015) Genetic polymorphisms associated with increased risk of developing chronic myelogenous leukemia. Oncotarget 6: 36269-36277.

29. Morgan MJ, Kim YS (2015) The serine threonine kinase RIP3: lost and found. BMB Reports 48: 303-312.

30. Koo GB, Morgan MJ, Lee DG, Kim WJ, Yoon JH, et al. (2015) Methylationdependent loss of RIP3 expression in cancer represses programmed necrosis in response to chemotherapeutics. Cell Res 25: 707-725.

31. Feng X, Song Q, Yu A, Tang $H$, Peng Z, et al. (2015) Receptor-interacting protein kinase 3 is a predictor of survival and plays a tumor suppressive role in colorectal cancer. Neoplasma 62: 592-601.

32. Bozec D, luga AC, Roda G, Dahan S, Yeretssian G (2016) Critical function of the necroptosis adaptor RIPK3 in protecting from intestinal tumorigenesis. Oncotarget 7: 46384-46400.

33. Colbert LE, Fisher SB, Hardy CW, Hall WA, Saka B, et al. (2013) Pronecrotic mixed lineage kinase domain-like protein expression is a prognostic biomarker in patients with early-stage resected pancreatic adenocarcinoma. Cancer 119 3148-3155.

34. He L, Peng K, Liu Y, Xiong J, Zhu FF (2013) Low expression of mixed lineage kinase domain-like protein is associated with poor prognosis in ovarian cancer patients. Onco Targets Ther 6: 1539-1543.

35. Ruan J, Mei L, Zhu Q, Shi G, Wang H (2015) Mixed lineage kinase domain-like protein is a prognostic biomarker for cervical squamous cell cancer. Int $\mathrm{J}$ Clin Exp Pathol 8:15035-15038.

36. Ertao Z, Jianhui C, Kang W, Zhijun Y, Hui W, et al. (2016) Prognostic value of mixed lineage kinase domain-like protein expression in the survival of patients with gastric cancer. Tumour Biol 37: 13679-13685

37. Li X, Guo J, Ding AP, Qi WW, Zhang PH, et al. (2017) Association of mixed lineage kinase domain-like protein expression with prognosis in patients with colon cancer. Technol Cancer Res Treat 16: 428-434.

38. Su Z, Yang Z, Xie L, DeWitt JP, Chen Y (2016) Cancer therapy in the necroptosis era. Cell Death Differ 23: 748-756.

39. Nehs MA, Lin Cl, Kozono DE, Whang EE, Cho NL, et al. (2011) Necroptosis is a novel mechanism of radiation-induced cell death in anaplastic thyroid and adrenocortical cancers. Surgery 150: 1032-1039.

40. Han W, Li L, Qiu S, Lu Q, Pan Q, et al. (2007) Shikonin circumvents cance drug resistance by induction of a necroptotic death. Mole Cancer Ther 6: 16411649

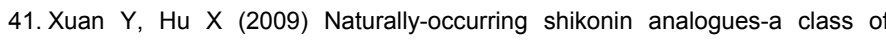
necroptotic inducers that circumvent cancer drug resistance. Cancer Lett 274 233-242.

42. Wong WW, Vince JE, Lalaoui N, Lawlor KE, Chau D, et al. (2014) clAPs and XIAP regulate myelopoiesis through cytokine production in an RIPK1- and RIPK3-dependent manner. Blood 123: 2562-2572.

43. Naijar M, Saleh D, Zelic M, Nogusa S, Shah S, et al. (2016) RIPK1 and RIPK3 kinases promote cell-death-independent inflammation by toll-like receptor 4 Immunity 45: 46-59.

44. Dey A, Mustafi SB, Saha S, Dwivedi SKD, Mukherjee P, Bhattacharya R (2016) Inhibition of BMI1 induces autophagy-mediated necroptosis. Autophagy 12 659-670. 